\title{
Addressing unmet needs in opiate dependence: supporting detoxification and advances in relapse prevention
}

\author{
Katherine Herlinger (1) \& Anne Lingford-Hughes
}

Katherine Herlinger, $\mathrm{MBChB}, \mathrm{BSc}$ (Hons), DipLaw, is a Clinical Research Fellow at Imperial College London, UK, currently conducting her $\mathrm{PhD}$ on the neurobiology of reward and emotional processing in opiate and alcohol dependence. Anne Lingford-Hughes, $\mathrm{MA}, \mathrm{PhD}$,

$\mathrm{MBChB}$, FRCPsych, is the Head of the Centre for Psychiatry and Professor of Addiction Biology at Imperial College London, UK. She is also a consultant psychiatrist with a particular interest in pharmacological treatments of alcohol problems and other substance addictions at Central North West London NHS Foundation

Trust, UK.

Correspondence Katherine Herlinger. Email: k.herlinger@imperial.ac.uk

First received 6 Jul 2020 Final revision 27 0ct 2020 Accepted 18 Nov 2020

\section{Copyright and usage}

(c) The Author(s), 2021. Published by Cambridge University Press on behalf of The Royal College of Psychiatrists. This is an Open Access article, distributed under the terms of the Creative Commons Attribution licence (http://creativecommons.org/ licenses/by/4.0/), which permits unrestricted re-use, distribution, and reproduction in any medium, provided the original work is properly cited.

\section{SUMMARY}

Despite record-breaking numbers of opiate related deaths in the UK in 2019, pharmacological management of opiate dependence has evolved little since the advent of methadone in 1965. Along with harm minimisation and psychosocial interventions, the mainstay of pharmacological treatment remains opioid substitution therapy (OST) using methadone or buprenorphine, with many patients receiving OST for many years. Even with these treatments, opiate users continue to face mortality risks 12 times higher than the general population, and emerging evidence suggests that individuals who remain on long-term OST present with a range of physical and cognitive impairments. Therefore, with a growing ageing opiate dependent population who would benefit from detoxification from OST, this article provides an overview of the current state of opiate dependence in clinical practice, explores the reasons why availability and acceptability of detoxification pathways are declining, and discusses emerging pharmacological therapies that could provide benefit in relapse prevention.

\section{LEARNING OBJECTIVES}

After reading this article you will be able to:

- contextualise the ongoing burden of opiate dependence

- better understand the needs of an ageing opioid dependent population

- recognise treatment gaps for opioid dependent individuals.

\section{KEYWORDS}

Opiate dependence; opioid substitution therapy; pharmacotherapy; detoxification; addiction.

Opioid related deaths are making global headlines of late. The USA is described as having an 'opioid epidemic' as opioid related drug deaths reach the highest recorded levels. America's heroin deaths increased five-fold between 2010 and 2017, equating to approximately 130 deaths daily and costing \$2.5 trillion between 2015 and 2018 (Council of Economic Advisers 2019). Meanwhile in Australia, opioid related deaths have almost doubled over the past 10 years, from 3.8 per 100000 in 2007 to 6.6 per 100000 in 2016 . The majority of these deaths (65\%) were due to prescription opioids alone (Roxburgh 2018).

In the UK, drug-related deaths are also at an all-time high. In 2018 England and Wales had the highest annual increase (16\%) in drug-related deaths since records began, the majority of these deaths (51\%) involving an opioid (Office for National Statistics 2019). These rates continue to be maintained, with drug-related deaths increasing by 0.8\% from 2018-2019 (Public Health England 2020a). Meanwhile, Scotland is reported to have had the highest number of drug-related deaths per million population of any country in the European Union (National Records of Scotland 2020).

Examining the statistics of drug trends in the UK indicates that opiate users currently make up the largest proportion of individuals accessing treatment in drug and alcohol services, $52 \%$ in 2019 2020 (Public Health England 2020a). More than half of these individuals (58\%) are over the age of 40 (Public Health England 2020b). Additionally, there has been a 34\% increase in those using opioids over the age of 35 between 2010 and 2017 , and $69 \%$ of those individuals started using heroin before 2001. This increase in individuals over the age of 35 in treatment is therefore not due to new users entering treatment but is consistent with an ageing opioid-dependent cohort (Public Health England 2019). Although this reflects the success of our treatment of opioid dependence, in that users are staying alive for longer, the deaths of middle-aged heroin users is one of the main drivers for the spike in drug-related deaths (Public Health England 2019). This dispels a commonly held belief that opioid related deaths usually occur from overdose in inexperienced users. 
Not only do these statistics indicate that the average age of opiate dependent individuals is on the rise, but additionally that the rate of abstinence is decreasing. Latest data from England shows that those with opiate dependence had the lowest rate of 'successful exits' (i.e. leaving treatment opioid-free) in 2019-2020 at 24\%, down from 28\% in 2015 and from a peak of $37 \%$ in 2011-2012. For comparison, the highest rate of 'successful exits' was for alcohol, at 59\% in 2018 (Public Health England 2020a).

Taken together, this reflects the trend that has been seen in research and medical treatment for opiate dependence, namely that following the focus on opioid substitution in the 1980s and 1990s, there has been limited scientific or clinical attention paid to either opiate detoxification (detox) or relapse prevention, with consequently limited innovations in recent decades.

In this article, we briefly describe the neurobiology of the opioid system and clinical management of opiate dependence. We then go on to discuss the decline in opiate detoxes, and the aspects of service provision and treatment we feel could be improved. Finally, we provide an update of current research being undertaken in this field.

A note on terminology: although often used interchangeably, the terms opiate and opioid have different meanings which are detailed in Box 1 below.

\section{The opioid system: an overview}

The opioid system in the brain is the main target for both endogenous and exogenous opioids (e.g. heroin, morphine). 'Good' effects from other substances of misuse, such as alcohol and amphetamines, have been shown to be associated with increases in endogenous opioids in the brain (Colasanti 2012; Mitchell 2012). There are three main types of opioid receptor - mu $(\mu)$, kappa $(\kappa)$ and delta $(\delta)$ - which are responsible for the range of effects of both exogenous and endogenous opioids (Table 1).

The mu-opioid receptor (MOR) is found in various brain regions involved in the reward circuitry, including the ventral tegmental area and ventral striatum. It is thought that effects elicited by this receptor are responsible for the misuse potential and reinforcing properties of opioids. The nucleus accumbens within the ventral striatum receives input from dopaminergic cell bodies in the ventral tegmental area via the mesolimbic pathway, while inhibitory gamma-aminobutyric acid (GABA) neurons project from the nucleus accumbens to the ventral palladium via the striatopallidial pathway (Nutt 2013). Opioids produce their effects in the ventral striatum directly by binding to MOR in the

ventral striatum or indirectly by binding to MOR on inhibitory GABA neurons, both of which increase dopaminergic neuronal firing.

The MOR is also found in abundance in brain regions that are implicated in emotion processing, motivation and impulsivity, which are cognitive processes shown to be dysregulated in those vulnerable to addiction and once addicted. Positron emission tomography (PET) studies have shown that individuals who report higher trait impulsivity scores have significantly higher MOR numbers in regions such as the amygdala, orbitofrontal cortex and other areas involved in motivated behaviour (Love 2009).

To maintain brain function and homeostasis in the presence of increasing circulating opioids, individuals will develop marked tolerance, namely reduced sensitivity and physical dependence, with repeated and prolonged opioid use. Such neurobiological adaptation is an expected response and seen with other drugs, such as benzodiazepines and alcohol. Thus, an individual can be dependent on a drug but not necessarily 'addicted', which is a complex behaviour including difficulty in controlling use and continuing despite harm. The latest changes to the DSM's diagnostic criteria for substance dependence (DSM-5; American Psychiatric Association 2013) reflect this (Box 2). Tolerance in opioid addiction commonly manifests itself as users requiring greater doses to produce the desired pleasant euphoric effects. Although this occurs rapidly, leading to reinforcement and increase of dosage and frequency of drug taking, tolerance for other effects of opioids, such as nausea and respiratory depression, can develop at different rates. For pupillary constriction, tolerance appears limited, thus making it clinically useful for assessing intoxication/withdrawal in addicts. Additionally, not all opioid agonists have the same mechanism and therefore cross-tolerance may be incomplete. This may help to explain why heroin use on top of opioid substitution therapy (e.g. methadone) can still result in fatal respiratory depression (Williams 2001).

BOX 1 Definitions: opiate versus opioid

An opiate is a psychoactive substance that is naturally derived from the flowering opium poppy plant (Papaver somniferum). Examples: morphine, codeine, thebaine. Historically the term 'opiate dependence' has been used to describe heroin dependence despite heroin being a semi-synthetic drug. In this article 'opiate dependence' is used to describe heroin dependence as a reflection of common practice.
An opioid is any substance (including opiates) that acts on opioid receptors to produce morphine-like effects. Originally this term was used only for synthetic opiates, but now it is used for the entire drug class, encompassing all naturally occurring, semi-synthetic and synthetic opiates. Examples: diacetylmorphine (heroin), endogenous opioids, oxycodone, fentanyl, hydromorphone, 
TABLE 1 Opioid receptor effects

\begin{tabular}{|cll|}
\hline Opioid receptor & $\begin{array}{l}\text { Associated } \\
\text { endogenous } \\
\text { opioids }\end{array}$ & Effects \\
\hline$\mu$ 'mu' (MOR) & $\beta$-endorphin & Euphoria \\
& & $\begin{array}{l}\text { Analgesia } \\
\text { Sedation }\end{array}$ \\
& & Respiratory \\
& & depression \\
& & Nausea and \\
& & vomiting \\
$\kappa$ 'kappa' (KOR) & Pupillary \\
& & constriction \\
& & Dysphoria \\
& & Stress \\
& Enkephalins $\mathrm{A}$ & Anxiolytic \\
& & Antidepressant \\
& & Analgesic \\
\hline
\end{tabular}

Development of physical dependence from regular, chronic use of opioids (including opioid analgesics) will also manifest itself as a withdrawal syndrome in the absence of opioids. Of particular relevance to treatment of opioid withdrawal is the 'noradrenergic storm'. At the MOR, opioids acutely decrease levels of cyclic adenosine monophosphate (cAMP), and with chronic use this is compensated for by up-regulation of the cAMP pathway so that that pathway returns to a normal level of function in the presence of a circulating opioid. When a circulating opioid is then removed, cAMP levels increase to far above normal levels. Functionally this occurs in the main noradrenaline-containing nucleus in the brain, the locus ceruleus, resulting in an increase in circulating noradrenaline (Nestler 2004). This accounts for some of the symptoms and signs observed in opioid withdrawal (Box 3). Understanding this underlying neurobiology has helped to inform appropriate pharmacological treatments for opioid withdrawal, such as the use of

BOX 2 DSM-5 and ICD-11 diagnostic criteria relating to substance use

Both the DSM and ICD have released revised versions in the past decade: DSM-5 (American Psychiatric Association 2013) and ICD-11 (World Health Organization 2019).

ICD-11 criteria relating to substance dependence largely remain unchanged from the previous version, separating 'harmful use' from 'dependence'. Conversely, DSM-5 has altered its nomenclature from 'addiction' and 'dependence' to 'substance use disorder', in part to mitigate the confusion surrounding these terms. 'Substance use disorder' is referred to as a continuum rather than distinct categories of 'abuse' and 'dependence'. alpha-2 adrenergic agonists in acute withdrawal, which will be discussed in more detail below.

There is evidence to suggest that chronic substance misuse has enduring effects on the endogenous opioid system that may contribute to the relapsing and remitting nature of substance dependence. PET studies using $\left[{ }^{11} \mathrm{C}\right]$-diprenorphine to label MOR, KOR and DOR receptors in opioid dependent individuals during early abstinence showed higher $\left[{ }^{11} \mathrm{C}\right]-$ diprenorphine binding in multiple brain regions compared with controls. This higher binding reflects an increase in opioid receptor availability due to either increased numbers of receptors or reduced circulating endogenous opioids. There was, however, no correlation between craving or withdrawal symptoms and opioid receptor availability (Williams 2007). Using the MOR-specific radiotracer $\left[{ }^{11} \mathrm{C}\right]$-carfentanil, higher MOR availability in the paralimbic brain regions following abrupt cessation of buprenorphine treatment has been shown in opioid dependent individuals; however, the relationship with craving and withdrawal was not examined (Zubieta 2000). Whether opioid receptor numbers and activity return to baseline levels following prolonged periods of abstinence from opioids remains unclear.

In keeping with findings of increased MOR binding in opioid dependence, PET studies have found that recently abstinent cocaine users also have higher MOR binding with $\left[{ }^{11} \mathrm{C}\right]$-carfentanil in

BOX 3 Common signs/symptoms of opioid withdrawal

Subjective

- Anxiety

- Irritability

- Insomnia

- Low mood

- Abdominal pain

- Craving

Objective

- Tremor

- Clammy skin

- Diarrhoea

- Nausea

- Yawning

- Coughing

- Sneezing

- Lacrimation

- Dilated pupils

- Increased heart rate

- Increased blood pressure 
the frontal, anterior cingulate and lateral temporal cortex, which was positively correlated with craving and predictive of relapse (Gorelick 2005, 2008). Similar findings have been reported for recently abstinent alcohol dependent individuals (Weerts 2011). Higher MOR availability has also been positively associated with craving for alcohol in this group (Heinz 2005). To investigate whether endogenous opioid levels were blunted in addiction our group used $\left[{ }^{11} \mathrm{C}\right]$-carfentanil to assess changes in levels following an oral D-amphetamine challenge. We showed that both pathological gamblers and abstinent alcoholics have blunted D-amphetamine-induced endogenous opioid release in various brain regions, including the nucleus accumbens, putamen and frontal lobe (Mick 2016; Turton 2020). Together this suggests that dysregulation of the endogenous opioid system is a feature of various substance and behavioural addictions.

\section{Treatment of opiate dependence}

Treatment for opiate dependence initially involves a phase of assessment and stabilisation using opioid substitution therapy (OST) and engagement with services. Following this, ongoing treatment can be broadly categorised as either harm reduction or abstinence oriented. Early access to medication is a key factor in engagement, but developing a strong therapeutic alliance is thought to be more important for long-term recovery (Moos 2007).

\section{Psychosocial interventions}

Specific psychosocial interventions are implemented on an individual basis, taking into account the goal of the treatment package, the person's needs and availability of trained staff and supervisors to deliver the intervention (Department of Health 2017). Initially the focus is on building a therapeutic relationship, identifying specific goals, identifying risks and creating a care plan with the patient to ensure that care can be delivered in a structured and cooperative manner. Motivational interviewing and contingency management are two of the recommended psychosocial interventions to assist in building a therapeutic relationship and helping patients to engage with services (Box 4). Other interventions, such as cognitive-behavioural therapy, cue exposure, social behaviour network therapy and couples therapy, may also provide benefit, depending on the circumstances.

Once a patient has become stable on OST, i.e. at a dose where they are comfortable and not using heroin 'on top' or misusing other drugs, treatment should focus on other recovery-related goals, such as abstinence. Ongoing engagement will include one-to-one keywork sessions in addition to a group recovery programme or mutual support group. In particular, treatment in combination with mutual support groups has been associated with better outcomes, improved functioning, improved long-term recovery rates and an overall reduction in cost to society (Department of Health 2017).

\section{Pharmacological interventions}

Pharmacological interventions for opiate dependence can be broadly categorised for use in either substitution, overdose, detoxification/withdrawal or relapse prevention.

\section{Opioid substitution therapy (OST)}

The underlying principle of OST is that administering an opioid medication with a longer half-life than

BOX 4 Recommended psychosocial interventions in opioid dependence

\section{Motivational interviewing}

In this empathetic style of counselling, special attention is focused on the patient's goals and repeated verbalisation of these. By encouraging the patient to explore their goals and the discrepancies between their actions and appropriate behaviours to help attain these goals, this communication is thought to bring about self-sufficient behavioural change (Miller 1983). There is a lack of studies investigating the effects of motivational interviewing in opiate dependence. However, a recent meta-analysis of randomised control trials (RCTs) using motivational interviewing for substance dependence has shown that, across all substances (excluding opiates, as no studies were identified), the intervention significantly improved outcomes at 6 months post-treatment, with effect sizes (Cohen's $d$ ) of 0.22 (95\% $\mathrm{Cl} 0.11-0.32$ ). This analysis did not find a significant effect immediately post-treatment (Sayegh 2017).

\section{Contingency management}

This intervention reinforces desired behaviours through incentives. An example would be regular clean urine drug samples in exchange for cash or voucher incentives, clinic privileges, stepping down of supervised consumption, etc. In particular, contingency management can improve completion of hepatitis $B$ vaccination in heroin dependence and is most commonly used for this (Weaver 2014). Results from a metaanalysis of RCTs examining the effect of contingency management on reducing substance use in substance dependence (pooled for all substances) indicated effect sizes (Cohen's $d$ ) of $0.46(95 \% \mathrm{Cl} 0.37-0.54)$ at the end of treatment. However, this appears to decrease over time: at 3 months post-intervention an effect size of 0.33 (95\% $\mathrm{Cl} 0.12-0.54)$ was reported, and there was no significant effect remaining after 6 months (Benishek 2014). 
illicit opioids will lead to complete cessation of illicit opioid use while also avoiding opioid withdrawal syndrome. Methadone, a MOR agonist, is the most widely used medication in opioid substitution. Its long half-life $(\sim 24 \mathrm{~h})$ compared with heroin (between 5 and 30 mins) means that it is less likely to result in withdrawal symptoms and cravings.

The other main medication used in OST is buprenorphine, which is a long-acting partial MOR agonist. This means that it never results in the same level of effects as a full agonist. Even at high doses buprenorphine results in less euphoria, sedation, respiratory depression and fatal overdose than methadone. Buprenorphine has high affinity at the MOR, so in the presence of other opioids it will antagonise their effects. Thus, if buprenorphine is taken when someone has an opioid agonist (e.g. heroin) in their system, withdrawal is precipitated. Conversely, in someone maintained on buprenorphine, MOR are occupied so that on-top heroin use results in no effect, making such illicit use less likely. It is worth noting that despite this, some individuals do use heroin on top of buprenorphine. Buprenorphine also has slow receptor dissociation kinetics, so in addition to reducing withdrawal symptoms and cravings in substitution, it allows for milder withdrawal during tapering and detoxification (Lutfy 2004). Although buprenorphine has a better safety profile than methadone, evidence indicates equal effectiveness in retaining patients in treatment (reviewed in Lingford-Hughes 2012).

\section{Overdose}

Naloxone is a fast-acting non-selective (i.e. MOR, KOR and DOR) opioid receptor antagonist, which has no intrinsic activity at any of these receptors. It is commonly used to reverse opioid-induced respiratory depression and is therefore lifesaving in such situations. In the UK, is now recommended that all opioid addicts have intramuscular naloxone available as take-home medication (Public Health England 2017). Police in Scotland are currently piloting officers carrying Naloxone, an approach that is also taken by several emergencies services in parts of the USA. Naloxone has also been combined with buprenorphine in a sublingual tablet, to reduce buprenorphine's misuse liability (naloxone is not active unless it is injected) (Ling 2010).

\section{Detoxification and withdrawal}

Opioid detoxification is defined as reducing opioid use over a predetermined period of time with the end goal of achieving abstinence. This is in contrast to the 'tapering' of an OST dose, which can occur over months or years. There is no evidence to suggest that detoxification is better tolerated or provides better outcomes from either methadone or buprenorphine, therefore an individual will usually reduce from their prescribed OST medication. In the UK, Department of Health guidance recommends a maximum 12 weeks for a community detox or 28 days for an in-patient setting (Department of Health 2017).

The alpha ${ }_{2}$-adrenergic agonist lofexidine may be used as an alternative in those with milder opiate dependence, those who do not want to use methadone or buprenorphine or want to detox in a shorter period of time. Alpha 2 -adrenergic agonists were developed from our knowledge about the contribution of an upregulated noradrenergic system or 'storm' to opioid withdrawal syndrome (see above; Nestler 2004). A typical course lasts between 7 and 10 days; therefore abstinence can be achieved faster than with detoxification with OST. Lofexidine was developed as an alternative to clonidine because of clonidine's hypotensive effects, which limited its use. Lofexidine has been available for decades in the UK, but it received a licence for use in the USA only in 2018. However, lofexidine was discontinued by the only UK manufacturer in 2018, and it is unclear when or if it will become available again. Additional pharmacological treatment of withdrawal is also generally given to attenuate other symptoms, such as Zdrugs for insomnia and loperamide for diarrhoea. In the UK there is now an increasing reliance on medications for symptomatic relief during planned opioid detox because of the discontinuation of lofexidine.

\section{Relapse prevention}

Despite the fact that opiate dependence is an enduring disorder characterised by periods of relapse, there are few abstinence aids or relapse prevention medications available. Naltrexone is a long-acting non-selective opioid antagonist and is currently the only licensed medication in the UK for relapse prevention, although it is rarely taken (see below). It should be given after complete detoxification from other opioids, as otherwise it can precipitate withdrawal symptoms (Lingford-Hughes 2012).

\section{Where have all the detoxes gone?}

As described above, OST in combination with psychosocial interventions remains the mainstay of treatment for opiate dependence. Undoubtedly this 'harm minimisation approach' using OST with psychosocial support has been highly effective in improving health and social functioning in opiate addiction (Lingford-Hughes 2012; Schuckit 2016; Sordo 2017). The improvements in infection rates and survival from HIV and hepatitis in particular 
have been transformative, and overall mortality remains significantly reduced for individuals who are receiving OST compared with those are not in treatment. In a meta-analysis by Sordo et al (2017), the out-to-in all-cause mortality rate ratio per 1000 person-years in and out of treatment was found to be 3.20 and 2.20 for methadone and buprenorphine respectively. However, in such studies, those who are not 'in treatment' are likely to have dropped out of treatment and to be still using rather than to have left treatment when they are abstinent and in recovery. Understandably then, improving accessibility to OST is a key approach in many parts of the world. However, a typical day in any addiction service will have opiate dependent individuals presenting for treatment and asking to 'get off all drugs'. It is striking that individuals do not present saying 'please prescribe me methadone for years', but that is what often happens.

In England, 94\% of opiate dependent individuals engage with services for at least 12 weeks, the highest proportion of all the addictions, which likely reflects the successful provision of OST. This is in direct contrast to opiate dependence having the lowest rate of 'successful exits' (i.e. leaving treatment opioid-free), as described above (other reasons for exiting treatment include dropping out, leaving the area, death and transferring to custody). Currently it appears that most detoxifications in England occur in the community. For 2019-2020, 8059 individuals receiving treatment for opiate dependence left either 'free of dependence' (840) or with no drug/alcohol use (7219), which would imply that these individuals either had a community detox or slow OST taper. There were only 1427 in-patient detoxifications reported. Given that 140599 individuals were recorded as receiving treatment for opiate dependence over that period, this suggests that only $5.73 \%$ overcame their dependence (Public Health England 2020b).

Contrast this with alcohol dependence, where providing a detox and relapse prevention pathway is generally seen as 'core business' for any addiction service. Individuals who are severely alcohol dependent generally require medically assisted withdrawal (i.e. detox) to prevent serious complications. This should be followed by a relapse prevention programme, which might include both pharmacological and psychosocial interventions. In a recent study of alcohol dependent individuals who were followed up 180 days after structured detoxification and rehabilitation, $69.6 \%$ had remained abstinent (Ledda 2019). The benefits of stopping drinking excessive amounts of alcohol are very clear (Nutt 2014), and the absence of an equivalent of OST for alcohol also helps alcohol dependent individuals to concentrate on detox and relapse prevention. This focus has resulted in the development and availability of a range of pharmacological options to support detox and relapse prevention in alcohol dependence (i.e. acamprosate, disulfiram, naltrexone and baclofen). The effectiveness of these adjuncts remains modest, reflecting adherence problems commonly seen with these patients and the complex nature of addiction biology (reviewed in Lingford-Hughes 2012).

Attention is now focusing on preventing and treating alcohol-related 'brain damage' as well as modulating cognitive processes underpinning addictive processes, such as reward, impulsivity and emotion processing. Similar approaches are being pursued for other addictions but rarely is such innovative activity applied to treating opioid addiction.

\section{Current challenges for detoxification and relapse prevention}

A range of social, environmental and biological factors are likely to be contributing to the low number of detoxes observed for opiate dependence. Owing to the complexity of the subject we have chosen to focus on three main issues that we believe are underpinning this decline: the culture of long-term OST prescribing, the 'outcomes' on the basis of which services are granted funding, and the lack of medications to support detox and relapse prevention. It is worth noting that several other factors are likely contributing to this, particularly in the UK, which has seen significant cuts in government funding to services as well as reduced in-patient services.

\section{OST dose and treatment length}

Guidance on the optimal length of maintenance OST has still not been standardised and remains person specific. In the UK a duration of 'months' is emphasised by the Department of Health guidelines (Department of Health 2017). However, in the USA guidance from the National Institute on Drug Abuse suggests that 12 months of methadone maintenance should be considered a minimum, and some patients may require years of treatment (National Institute on Drug Abuse 2020). In practice, many people remain on OST for many months or even years and have multiple episodes of OST of such duration. In one meta-analysis $51 \%$ of the cohorts studied had been receiving methadone treatment for 2 years or more (McCowan 2009).

A further matter for consideration is optimisation of an individual's maintenance OST dose. Department of Health guidance in the UK recommends a methadone dose of between 60 and 120 mg daily (Department of Health 2017). This dose 
is based on randomised controlled trials mostly published in the 1990s which indicated that higher doses of methadone were more effective at retaining opiate dependent individuals in treatment and reducing on-top heroin use (Faggiano 2003). Despite this evidence and clinical guidelines, it is common to see lower doses prescribed in clinical practice. In one longitudinal study $85 \%$ of the cohort were receiving less than the recommended $60 \mathrm{mg}$ minimum methadone maintenance dose (McCowan 2009). A report from the Advisory Council on the Misuse of Drugs (ACMD) in 2015 cited several factors that contribute to this phenomenon, including prescriber factors such as reluctance due to moral reasons and fear of diversion. The dangers of diversion are particularly prominent for higher OST doses, where diversion could be fatal, and supervision of consumption is often employed to minimise diversion risk. Conversely, patient factors include concerns about side-effects of high doses, concern about never being able to 'come off', and being able to continue to use heroin on top of the OST dose. The report also suggests that slow reduction regimes may in fact be long-term underdosing, leading to on-top heroin use and longer periods of suboptimal OST.

Although studies conducted in the 1990s indicated that increased length of OST is associated with favourable outcomes, the effects of chronic opioid exposure on physical health perhaps were less considered. In a longitudinal study of opioid and other drug users, the most common cause of death in the opioid users was cardiovascular incident (including ischaemic heart disease and cerebrovascular disease), particularly in those aged over 55 years. This was interestingly higher than for users of central nervous system stimulants (Stenbacka 2010). Methadone and opioid painkillers have been associated with sleep-related problems such as sleep-disordered breathing, central sleep apnoea and obstructive sleep apnoea, all of which contribute to lethal disorders of breathing during sleep (Hassamal 2016). Chronic opioid analgesia use has also been associated with wakeful ataxic breathing, and again increasing age appears to be a risk factor (Walker 2007).

Evidence also suggests that chronic opioid use has enduring neurocognitive effects. A meta-analysis of assessing decision-making in opiate users has shown that chronic users exhibited consistent deficits on a range of decision-making tasks, and that these deficits were still present after cessation of use (Biernacki 2016). In a recent meta-analysis conducted by Baldacchino et al (2017), methadonemaintained individuals were found to have neurocognitive deficits in working memory, attention, cognitive flexibility and other areas compared with controls. This highlights that these deficits are not exclusive for illicit opioids, but affect those on OST and prescribed analgesia. Impairment in these domains can fuel ongoing poor decision-making and consumption behaviours in individuals, which may affect continuing drug use and relapse.

Finally, overall mortality is increased for individuals taking methadone who have coexisting conditions such as cardiovascular disease, respiratory diseases and diabetes (McCowan 2009). This is of increasing concern for the ageing opiatedependent population, who are likely to have comorbidities as a result of their drug use and lifestyle. This concern has been specifically addressed by the ACMD and Department of Health: the latter mentioned the 'ageing cohort of those with heroin dependence in treatment needing a focus on improving their morbidity and mortality' as a reason for having updated the 2007 guideline (Department of Health 2017). It has more recently been highlighted as a key finding in Dame Carol Black's independent review of drugs, which was commissioned by the UK government in 2019 (Black 2020). However, owing to the lack of alternative options for treating opiate dependence there continues to be a heavy reliance on long-term OST despite the fact that detoxification may be more appropriate for many individuals.

\section{Definitions of outcomes}

Achieving abstinence or gaining substantial recovery in terms of physical and mental health, employment or other social aspects may not be a realistic goal for some at any time during their treatment, and receiving long-term OST is likely to be the most appropriate treatment alongside psychosocial support for such individuals. Understandably, therefore, outcome data often focus on 'numbers in treatment', so engaging and retaining individuals is used as an indicator of how well services are performing.

It is therefore reasonable to propose that the focus on harm minimisation, OST and importance of 'good outcomes' for services in obtaining and sustaining their funding has contributed to reduced provision and reduced acceptability of opioid detoxification to services and opiatedependent individuals. This is not to advocate detoxification and abstinence (i.e. no use of illicit or prescribed opioids) as a gold-standard goal of treatment or one that should be achieved by everyone; rather, it should be one of a range of treatment options for those who present wanting to achieve abstinence. A recent report from the European Monitoring Centre for Drugs and Drug Addiction (EMCDDA) included a case study from England which stated that 'our focus was getting people stable on OST, 
retaining them and preventing harm, but we lacked focus on long-term goals' and 'staff were professional and busy but they were risk averse and not as optimistic as they could have been about service users' long-term outcomes' (2017: p. 11).

Debate concerning how to define outcomes for addiction services is not new and is seen in all areas of the field, from service provision and policy to research. The EMCDDA has previously noted that there appears to be a lack of consensus on treatment goals and that there is a need to clarify main outcomes and appropriate measures of these (Weissing 2014). This confusion reflects the complexity and diversity of the patients treated. However, just as abstinence rates should not be taken as the gold standard of successful treatment, so 'numbers in treatment' should not be the only measure of successful service provision.

\section{Lack of medications to support detoxification and abstinence}

Detoxification from all opioids, including those prescribed in OST, remains difficult for patients, and now that lofexidine is no longer available in the UK, psychosocial support and reducing OST with symptomatic medication are the only remaining interventions that can be offered. In light of this, one might expect a focus on identifying novel therapeutic targets for opioid dependence; for instance our group has found that neurokinin-1 (NK-1) receptor antagonism modulates brain responses during tasks relevant to addiction consistent with its potential to treat opioid and other addictions (Paterson 2017; Volkow 2018). However, there is currently limited investigation of alternative medications for relapse prevention, with almost all trials on clinicaltrials.gov involving an opioid antagonist and/ or remaining on OST with the goal of no 'on-top' use rather than abstinence from all opioids including OST. For example, the Prescription Opioid Addiction Treatment Study (NCT00316277) was a clinical trial completed in 2013 using a buprenorphine/naloxone treatment regime for prescription opioiddependent individuals. This heavy reliance on substitution has become commonplace in opioid dependence, but compare this to alcohol treatment, where in improving treatment, it is unlikely that you would compare a substitute (e.g. alcohol or benzodiazepines) with relapse prevention (e.g. disulfiram, naltrexone).

Concern about risk of fatal relapse to opiate use is also a powerful message to encourage continued use of OST. The opioid antagonist naltrexone is the only medication for relapse prevention licensed in the UK and it is based on its ability to block access of consumed opiates to the opioid receptors. Despite the availability of naltrexone for relapse prevention, poor adherence is commonplace, with most dependent opiate users returning to their drug use. Naltrexone is also often not offered in clinical practice; one review found that naltrexone was encouraged in only a minority of community buprenorphine detox studies, with many reports not mentioning its use (Dunn 2011). A recent review of treatment strategies to manage opioid withdrawal and initiate treatment with naltrexone concluded that 'treatment strategies to manage opioid withdrawal have evolved, but many patients with OUD [opioid use disorder] do not receive medication for the prevention of relapse' (Bisaga 2018).

To account for poor adherence to naltrexone, depot preparations have been developed but they are currently licensed only in the USA. Clinical trials in the USA are also underway for a naltrexone implant, whereas in Australia naltrexone implants are unlicensed but currently legal. Initial findings from Australia comparing crude mortality rates in opioiddependent individuals treated with the naltrexone implant have shown no overall improvement compared with methadone or buprenorphine. There was, however, a significant decrease in the crude mortality rate during the induction (first 28 days) period for the naltrexone implant when compared with methadone (Kelty 2019). Although these preparations might confer some therapeutic benefit, with only one pharmacological intervention available as an abstinence aid, the unmet needs of individuals who wish to detox and then remain abstinent from opiates are obvious. Addressing this gap would not only have a positive impact on the lives of patients, but also reduce the economic burden of opiate dependence on society.

\section{Current and future directions}

Although psychosocial approaches are the mainstay of treating addiction, we have seen how adjunctive medication can play a vital role in preventing complications, reducing illicit drug use, supporting abstinence or protecting the individual (e.g. an opioid antagonist such as naloxone in opioid overdose). Understanding the underlying neurobiology of addiction is therefore vital to inform developments, particularly of adjunctive medication, to improve outcomes. In particular, there is evidence to show that drug addiction is associated with dysregulation in several neural networks involved in reward, stress and inhibitory control. Pharmacological treatments that might modulate any dysregulation of these systems have been investigated to improve outcomes in alcohol and cocaine addiction but only recently has this approach been applied to opiate addiction.

The National Institute on Drug Abuse recently published its top ten 'medication development 
MCO answers

$\begin{array}{lllll}1 \mathrm{e} & 2 \mathrm{a} & 3 \mathrm{c} & 4 \mathrm{~d} & 5 \mathrm{~b}\end{array}$ priorities in response to the opioid crisis': orexin, kappa opioid, nociceptin opioid peptide, GABA-B, muscarinic M5, glutamate (AMPA and mGluR2/ 3), ghrelin, dopamine $\mathrm{D}_{3}$ and cannabinoid (Rasmussen 2019). This is a welcome development and focuses on 'the development of novel therapeutics to treat opioid overdose and OUD [opioid use disorder] in the near term' (emphasis as in original). Thus, medications exist for these targets but are licensed for another indication, so 'repurposing' such medications is already underway. It is often fortunate that once a neurobiological target has been identified, a medication with appropriate pharmacological profile is already available to speed up translation, again highlighting the importance of understanding underlying neurobiology.

\section{Final note from the authors}

At the time of writing, we are in the midst of the COVID-19 pandemic and witnessing first-hand the effects this is having on patients and service delivery. As services are being restructured to minimise patient contact and therefore reduce the spread of the coronavirus, in the UK opioid detoxes were initially suspended. Community detoxes are now slowly being reintroduced, but services are seeing increased waiting times for in-patient detoxes. There is an emphasis on starting or switching patients from methadone to buprenorphine where possible, with minimal visits to clinic during titration to minimise clinical contact further. However, many patients are still preferring treatment with methadone. OST is being delivered largely 'unsupervised', to help people to stay at home, particularly those who are clinically vulnerable, and not to overwhelm pharmacy capacity. Access to the supervised OST we have become so reliant on is becoming increasingly difficult. As we start to recover from this pandemic, there will be an inevitable backlog of issues that will need to be addressed for this population and the need for better detoxes and relapse prevention will be even more pressing.

\section{Supplementary material}

Supplementary material is available online at https://doi.org/10.1192/bja.2020.98.

\section{Author contributions}

K.H. has written this article as first author. A.L.-H. contributed to the writing and review of this article.

\section{Funding}

This work received no specific grant from any funding agency, commercial or not-for-profit sectors.

\section{Declaration of interest}

K.H. is currently being funded by the Medical Research Council, through the MRC Addiction Research Clinical Training Programme (MARC), to complete her PhD. A.L.-H. has received grants from GSK during the conduct of the submitted work; outside the submitted work, she has received grants and personal fees from Lundbeck; personal fees from Silence Therapeutics, Janssen-Cilag, Pfizer, Sanofi-Aventis; other fees from Opiant Pharmaceuticals and Lightlake Therapeutics; and support for a $\mathrm{PhD}$ from Alcarelle.

ICMJE forms are in the supplementary material, available online at https://doi.org/10.1192/bja. 2020.98 .

\section{References}

Advisory Council on the Misuse of Drugs (2015) How Can Opioid Substitution Therapy (and Drug Treatment and Recovery Systems) Be Optimised to Maximise Recovery Outcomes for Service Users? ACMD.

American Psychiatric Association (2013) Diagnostic and Statistical Manual of Mental Disorders (5th edn) (DSM-5). American Psychiatric Publishing.

Baldacchino A, Armanyous M, Balfour DJ, et al (2017) Neuropsychological functioning and chronic methadone use: a systematic review and metaanalysis. Neuroscience and Biobehavioral Reviews, 73: 23-38.

Benishek LA, Dugosh KL, Kirby KC, et al (2014) Prize-based contingency management for the treatment of substance abusers: a meta-analysis. Addiction, 109: 1426-36.

Biernacki K, McLennan SN, Terrett G, et al (2016) Decision-making ability in current and past users of opiates: a meta-analysis. Neuroscience and Biobehavioral Reviews, 71: 342-351.

Bisaga A, Mannelli P, Sullivan MA, et al (2018) Antagonists in the medical management of opioid use disorders: historical and existing treatment strategies. American Journal on Addictions, 27: 177-87.

Black DC (2020) Review of Drugs: Executive Summary. GOV.UK.

Colasanti A, Searle GE, Long CJ, et al (2012) Endogenous opioid release in the human brain reward system induced by acute amphetamine administration. Biological Psychiatry, 72: 371-7.

Council of Economic Advisers (2019) The Role of the Opioid Prices in the Evolving Opioid Crisis. Council of Economic Advisers.

Department of Health (2017) Drug Misuse and Dependence: UK Guidelines on Clinical Management. Prepared by Clinical Guidelines on Drug Misuse and Dependence Update 2017 Independent Expert Working Group.

Dunn KE, Sigmon SC, Strain EC, et al (2011) The association between outpatient buprenorphine detoxification duration and clinical treatment outcomes: a review. Drug and Alcohol Dependence, 119: 1-9.

European Monitoring Centre for Drugs and Drug Addiction (2017) Recovery, Reintegration, Abstinence, Harm Reduction: The Role of Different Goals within Drug Treatment in the European Context. EMCDDA.

Faggiano F, Vigna-Taglianti F, Versino E, et al (2003) Methadone maintenance at different dosages for opioid dependence. Cochrane Database of Systematic Reviews, 3: CD002208.

Gorelick DA, Kim YK, Bencherif B, et al (2005) Imaging brain mu-opioid receptors in abstinent cocaine users: time course and relation to cocaine craving. Biological Psychiatry, 57: 1573-82.

Gorelick DA, Kim YK, Bencherif B, et al (2008) Brain mu-opioid receptor binding: relationship to relapse to cocaine use after monitored abstinence. Psychopharmacology, 200: 475-86

Hassamal S, Miotto K, Wang T, et al (2016) A narrative review: the effects of opioids on sleep disordered breathing in chronic pain patients and 
methadone maintained patients. American Journal on Addictions, 25 . 452-65.

Heinz A, Reimold M, Wrase J, et al (2005) Correlation of stable elevations in striatal $\mu$-opioid receptor availability in detoxified alcoholic patients with alcohol craving: a positron emission tomography study using carbon 11-labeled carfentanil. Archives of General Psychiatry, 62: 57-64.

Kelty E, Joyce D, Hulse G (2019) A retrospective cohort study of mortality rates in patients with an opioid use disorder treated with implant naltrexone, oral methadone or sublingual buprenorphine. American Journal of Drug and Alcohol Abuse, 45: 285-91.

Ledda R, Battagliese G, Attilia F, et al (2019) Drop-out, relapse and abstinence in a cohort of alcoholic people under detoxification. Physiology \& Behavior, 198: 67-75

Ling W, Jacobs P, Hillhouse M, et al (2010) From research to the real world: buprenorphine in the decade of the Clinical Trials Network. Journal of Substance Abuse Treatment, 38(suppl 1): S53-60.

Lingford-Hughes AR, Welch S, Peters L, et al (2012) BAP updated guidelines: evidence-based guidelines for the pharmacological management of substance abuse, harmful use, addiction and comorbidity: recommendations from BAP. Journal of Psychopharmacology, 26: 899-952.

Love TM, Stohler CS, Zubieta JK (2009) Positron emission tomography measures of endogenous opioid neurotransmission and impulsiveness traits in humans. Archives of General Psychiatry, 66: 1124-34.

Lutfy K, Cowan A (2004) Buprenorphine: a unique drug with complex pharmacology. Current Neuropharmacology, 2: 395-402.

McCowan C, Kidd B, Fahey T (2009) Factors associated with mortality in Scottish patients receiving methadone in primary care: retrospective cohort study. BMJ, 338: b2225.

Mick I, Myers J, Ramos AC, et al (2016) Blunted endogenous opioid release following an oral amphetamine challenge in pathological gamblers. Neuropsychopharmacology, 41: 1742-50

Miller WR (1983) Motivational interviewing with problem drinkers. Behavioural and Cognitive Psychotherapy, 11: 147-72.

Mitchell JM, O'Neil JP, Janabi M, et al (2012) Alcohol consumption induces endogenous opioid release in the human orbitofrontal cortex and nucleus accumbens. Science Translational Medicine, 4(116): 116ra6.

Moos RH (2007) Theory-based active ingredients of effective treatments for substance use disorders. Drug and Alcohol Dependence, 88: 109-21.

National Institute on Drug Abuse (2020) Principles of Effective Treatment: A Research-Based Guide (3rd edn). NIDA.

National Records of Scotland (2020) Drug-Related Deaths in Scotland in 2019. National Records of Scotland. (https://www.nrscotland gov.uk/ statistics-and-data/statistics/statistics-by-theme/vital-events/deaths/drugrelated-deaths-in-scotland/2019)

Nestler EJ (2004) Historical review: molecular and cellular mechanisms of opiate and cocaine addiction. Trends in Pharmacological Sciences, 25: 210-8.

Nutt D, Nestor L (2013) Addiction. Oxford University Press.

Nutt DJ, Rehm J (2014) Doing it by numbers: a simple approach to reducing the harms of alcohol. Journal of Psychopharmacology, 28: 3-7.

Office for National Statistics (2019) Deaths Related to Drug Poisoning in England and Wales 2018 Registrations (Statistical Bulletin). ONS

Paterson LM, McGonigle J, Giribaldi B, et al (2017) NK1 antagonism attenuates blunted response to monetary reward anticipation in abstinent alcohol dependence. Journal of Psychopharmacology, 31: A122.

Public Health England (2017) Take-Home Naloxone for Opioid Overdose in People Who Use Drugs. PHE.
Public Health England (2019) Adult Substance Misuse Treatment Statistics 2018 to 2019: Report. PHE (https://www.gov.uk/government/publications/ substance-misuse-treatment-for-adults-statistics-2018-to-2019/adultsubstance-misuse-treatment-statistics-2018-to-2019-report).

Public Health England (2020a) Adult Substance Misuse Treatment Statistics 2019 to 2020: Report. PHE (https://www.gov.uk/government/publications/ substance-misuse-treatment-for-adults-statistics-2019-to-2020/adult-substancemisuse-treatment-statistics-2019-to-2020-report|

Public Health England (2020b) Adult Substance Misuse Treatment Statistics 2019 to 2020: Data Tables. PHE (https://www. gov.uk/government/statistics/ substance-misuse-treatment-for-adults-statistics-2019-to-2020).

Rasmussen K, White DA, Acri JB (2019) NIDA's medication development priorities in response to the Opioid Crisis: ten most wanted. Neuropsychopharmacology, 44: 657-9.

Roxburgh A, Dobbins T, Degenhardt L, et al (2018) Opioid-, amphetamine-, and cocaine-induced deaths in Australia: August 2018. National Drug and Alcohol Research Centre, UNSW.

Sayegh CS, Huey SJ, Zara EJ, et al (2017) Follow-up treatment effects of contingency management and motivational interviewing on substance use: a meta-analysis. Psychology of Addictive Behaviors, 31: 403-14.

Schuckit MA (2016) Treatment of opioid-use disorders. New England Journal of Medicine, 375: 357-68

Sordo L, Barrio G, Bravo MJ, et al (2017) Mortality risk during and after opioid substitution treatment: systematic review and meta-analysis of cohort studies. BMJ, 357: j1550.

Stenbacka M, Leifman A, Romelsjo A (2010) Mortality and cause of death among 1705 illicit drug users: a 37 year follow up. Drug and Alcohol Review, 29: 21-7.

Turton S, Myers JF, Mick I, et al (2020) Blunted endogenous opioid release following an oral dexamphetamine challenge in abstinent alcoholdependent individuals. Molecular Psychiatry, 25: 1749-58.

Volkow ND, Boyle M (2018) Neuroscience of addiction: relevance to prevention and treatment. American Journal of Psychiatry, 175: 729-40.

Walker JM, Farney RJ, Rhondeau SM, et al (2007) Chronic opioid use is a risk factor for the development of central sleep apnea and ataxic breathing. Journal of Clinical Sleep Medicine, 3: 455-61.

Weaver T, Metrebian N, Hellier J, et al (2014) Use of contingency management incentives to improve completion of hepatitis $B$ vaccination in people undergoing treatment for heroin dependence: a cluster randomised trial. Lancet, 384: 153-3.

Weerts EM, Wand GS, Kuwabara H, et al (2011) Positron emission tomography imaging of mu- and delta-opioid receptor binding in alcoholdependent and healthy control subjects. Alcoholism, Clinical and Experimental Research, 35: 2162-73.

Weissing L, Ferri M (2014) What Are the Outcomes for Assessing Drug Treatment? A Review of Long-Term Observational Studies on the Treatments of Opioid Dependence. EMCDDA

Williams JT, Christie MJ, Manzoni 0 (2001) Cellular and synaptic adaptations mediating opioid dependence. Physiological Reviews, 81: 299-343.

Williams TM, Daglish MRC, Lingford-Hughes A, et al (2007) Brain opioid receptor binding in early abstinence from opioid dependence: positron emission tomography study. British Journal of Psychiatry, 191: 63-69.

World Health Organization (2019) International Classification of Diseases 11th Revision. Available from: https://icd.who.int/en.

Zubieta J-K, Greenwald MK, Lombardi U, et al (2000) Buprenorphineinduced changes in mu-opioid receptor availability in male heroin-dependent volunteers: a preliminary study. Neuropsychopharmacology, 23: 326-34. 


\section{MCOs}

Select the single best option for each question stem

1 Opioids produce their rewarding effects by binding directly to $\mathrm{mu}(\mu)$ opioid receptors in the:

a nucleus accumbens

b ventral palladium

c orbitofrontal cortex

d amygdala

e ventral striatum.

2 Symptoms of opioid withdrawal do not include:

a decreased heart rate

b lacrimation

c sneezing

d coughing

e increased blood pressure.
3 As regards naltrexone and opioid dependence:

a naltrexone is acts on mu- and delta-opioid receptors

b naltrexone can be given during opioid detoxification

c naltrexone is the only licensed medication for relapse prevention in the UK

d naltrexone has no intrinsic activity at opioid receptors

e naltrexone has historically good clinical adherence.

4 Chronic opioid use has not been associated with:

a ischaemic heart disease

b obstructive sleep apnoea

c deficits in working memory

d type 2 diabetes mellitus

e deficits in attention.
5 Contingency management is a recommended psychosocial intervention in opioid dependence that is best defined as:

a an empathetic style of goal-directed interviewing

b an intervention that reinforces desired behaviours through incentives

c an intervention that defines a concrete plan in the event of relapse

$\mathrm{d}$ an intervention that involves identifying and challenging unhelpful behaviours

e an empathetic interview style that aims to uncover unconscious thought underlying behaviour. 